\title{
Alcohol and the heart: to abstain or not to abstain?
}

\author{
Rajesh Movva, MD, MRCP \\ Albert Einstein Medical Center, Philadelphia1; \\ Vincent M. Figueredo, M.D. \\ Thomas Jefferson University
}

\section{Follow this and additional works at: https://jdc.jefferson.edu/cardiologyfp \\ Part of the Cardiology Commons \\ Let us know how access to this document benefits you}

\section{Recommended Citation}

Movva, MD, MRCP, Rajesh and Figueredo, M.D., Vincent M., "Alcohol and the heart: to abstain or not to abstain?" (2013). Division of Cardiology Faculty Papers. Paper 28.

https://jdc.jefferson.edu/cardiologyfp/28

This Article is brought to you for free and open access by the Jefferson Digital Commons. The Jefferson Digital Commons is a service of Thomas Jefferson University's Center for Teaching and Learning (CTL). The Commons is a showcase for Jefferson books and journals, peer-reviewed scholarly publications, unique historical collections from the University archives, and teaching tools. The Jefferson Digital Commons allows researchers and interested readers anywhere in the world to learn about and keep up to date with Jefferson scholarship. This article has been accepted for inclusion in Division of Cardiology Faculty Papers by an authorized administrator of the Jefferson Digital Commons. For more information, please contact: JeffersonDigitalCommons@jefferson.edu. 


\section{As submitted to: \\ International Journal of Cardiology}

And later published as

Alcohol and the heart: To abstain or not to abstain?

April 2013, Volume 164, Issue 3, pp. 267-76

DOI: $10.1016 /$ j.ijcard.2012.01.030

Rajesh Movva MD ${ }^{1}$, MRCP; Vincent M. Figueredo MD ${ }^{1,2}$

Author for Correspondence:

Vincent M. Figueredo MD, FACC, FAHA, FASE

Director, Cardiovascular Fellowship program

Associate Professor, Thomas Jefferson University

Einstein Heart and Vascular Institute

Albert Einstein Medical Center

5501 Old York Road

Philadelphia PA 19141

E-mail: FigueredoV@einstein.edu

Phone \# 215-456-8819; Fax \# 215-456-3533.

Author affiliations: Albert Einstein Medical Center, Philadelphia ${ }^{1}$; Jefferson Medical College, Thomas Jefferson University, Philadelphia. ${ }^{2}$ 
Key words: Alcohol, Coronary artery disease, Heart failure, Cardiomyopathy, Coronary heart disease.

\section{Abstract}

Alcohol has been consumed by most societies over the last 7000 years. Abraham Lincoln said "It has long been recognized that the problems with alcohol relate not to the use of a bad thing, but to the abuse of a good thing." Light to moderate alcohol consumption reduces the incidence of coronary heart disease (CHD), ischemic stroke, peripheral arterial disease, CHD mortality, and all-cause mortality, especially in the western populations. However, heavy alcohol consumption is detrimental causing cardiomyopathy, cardiac arrhythmias, hepatic cirrhosis, pancreatitis, and hemorrhagic stroke. In this article, we review the effects of alcohol on CHD, individual cardiovascular risk factors, cardiomyopathy, and cardiac arrhythmias, including the most recent evidence of the effects of alcohol on CHD.

\section{Alcohol and Global Burden of Disease}

An estimated $3.8 \%$ of all global deaths are attributable to the detrimental effects of alcohol consumption.[1] Disease burden and disability are closely related to the volume of alcohol consumption.[1] Alcohol dependence is a major health and social issue affecting 4 to $5 \%$ of the United States population at any given time,[2] with a lifetime prevalence of $12.5 \%$.[3] In the United States, approximately $30 \%$ of those who drink alcohol do so excessively. Excessive drinking is the third leading preventable cause of death, accounting for 79,000 deaths annually, and resulting in approximately 2.4 million years of potential life lost.[4,5] Heavy drinkers (> 6 drinks) had a doubled mortality rate, and consumption of 3 to 5 drinks was associated with a 50\% higher mortality rate.[6] 
However, moderate alcohol consumption is protective against CHD, [7] with higher cardiovascular event rates occurring among abstainers and heavy drinkers. There is a plethora of epidemiological evidence, demonstrating a J- or U-shaped association between alcohol consumption and all-cause mortality (Figure 1),[8] as well as cardiovascular morbidity and mortality (Figure 2).[9] However, this hypothesis was challenged by a meta-analysis that showed a lack of benefit of alcohol in preventing CHD.[10] In addition, ethnicity, gender, type of alcoholic beverage, and pattern of alcohol intake, may also influence the relation between alcohol and CHD.

\section{Epidemiological Studies Showing a Favorable Effect of Alcohol on the}

\section{Heart}

Ethanol consumption showed a strong U-shaped relationship with CHD mortality for male nonsmokers, and heavy smokers, irrespective of beverage type, and for female smokers, after a 24-year follow-up. In nonsmokers, beer and wine were associated with greater reductions in CHD mortality than spirits.[11] Light and moderate alcohol consumption were inversely associated with cardiovascular mortality over a 25 year period, even when compared with lifetime abstainers.[12]

In a pooled analysis of 8 prospective studies from North America and Europe, including 192,067 women and 74,919 men free of cardiovascular diseases and diabetes, there was an inverse association between alcohol and risk of CHD in all age groups.[13] In the Health Professionals Follow-up Study, moderate alcohol intake was associated with lower risk for myocardial infarction (MI) in men free of major illness, during 16 years of 
follow-up of 8867 physicians.[14] A prospective cohort study of 64,597 Chinese men aged $\geq 40$ years, who were free of clinical CHD, showed alcohol consumption was associated with a lower risk of MI and CHD after 494,084 person-years follow-up.[15] Maintaining alcohol consumption below 46g/day in men and $23 \mathrm{~g}$ /day in women appears to minimize the risks of mortality in a Japanese population, based on a pooled analysis of six ongoing large-scale cohort studies $(n=309,082)$. There was a J- or U-shaped association for the risk of total and major causes of mortality in men, and the risk of total and heart disease mortality in women.[16] A J-shaped relation between alcohol consumption and incident cardiovascular disease (CVD), and total and CVD deaths was found during a mean follow up of 12.2 years in 26,399 women.[17] Compared with abstainers, alcohol intake of 5 to $14.9 \mathrm{~g} / \mathrm{d}$ was associated with $26 \%, 35 \%$, and $51 \%$ lower risk of CVD events, total death, and CVD death, respectively, in a multivariable model.[17] In a Japanese study of 34,776 men and 48,906 women aged 40 to 79 years, followed-up for a median duration of 14.2 years, heavy alcohol consumption was associated with increased CVD mortality in men, and CHD in women, whereas light-tomoderate drinking was associated with reduced CVD mortality in both sexes.[18]

In the Nurses' Health study of 87,526 female nurses aged 34 to 59 years, moderate alcohol consumption decreased the risks of CHD during 334,382 person-years of followup, compared with nondrinkers.[7] In the Women's Health Initiative (WHI) comprising 10,576 black and 105,610 white postmenopausal women free of cardiovascular disease, moderate drinking was associated with a lower risk of total mortality among Caucasian women during a mean follow-up of 8 years, regardless of hypertensive status, but only in 
hypertensive African-American women.[19] In the Swedish Women's Lifestyle and Health Study of 47,921 women aged 30-49 years, a significant inverse association was found between light to moderate drinking and total mortality. This was predominantly driven by reduction in cardiovascular mortality, during 713,295 person-years followup.[20] In the Women's Health Initiative Observational Study, during the 22,546 personyears of follow-up, both frequency and quantity of alcohol intake were inversely associated with the risk of developing CHD, irrespective of the beverage type.[21] Moderate alcohol consumption in postmenopausal women may have a benefit on CHD, irrespective of their diabetic status.[21] In the Nurses' Health Study, moderate alcohol consumption was associated with reduced CHD risk in women with diabetes, during 39,092 person-years of follow-up.[22] Moderate alcohol consumption was protective against coronary atherosclerosis progression demonstrated by quantitative coronary angiography in the Stockholm Female Coronary Risk Angiographic Study.[23]

Moderate alcohol consumption also reduces mortality in patients with documented CVD. Light to moderate alcohol consumption (5 to $25 \mathrm{~g} /$ day) was associated with a lower incidence of CVD events and all-cause mortality in a recent meta-analysis of 8 prospective studies including 16,351 subjects with history of CVD. J-shaped pooled curves were observed with both CVD and all-cause mortality.[9] In patients with clinically manifest vascular disease or diabetes $(n=5447)$ from the Second Manifestations of Arterial Disease (SMART) study, moderate alcohol consumption (1-2 drinks/day) was not only associated with a reduced risk of vascular and all-cause death, but also with reduced risks of non-fatal events from CHD, stroke and amputations.[24] In 
the Prospective Epidemiological Study of Myocardial Infarction (PRIME), in Northern Ireland and France, regular and moderate alcohol intake was associated with a low risk of CHD, whereas the binge drinking pattern conferred a higher risk. [25] Only wine drinking was associated with a lower risk of hard coronary events.[25] Moderate alcohol intake was inversely associated with CHD in hypertensive men in the Physicians' Health Study, and Health Professionals Follow-Up Study, after 26- and 16-year follow-ups, respectively.[26, 27]

Moderate alcohol consumption in the year prior to acute MI was associated with reduced total and CV mortality following infarction, irrespective of gender and beverage type,[28] and a significant reduction in cardiovascular complications in survivors of acute MI.[29] In the Stockholm Heart Epidemiology Program (SHEEP) study, moderate alcohol drinking was beneficial following acute MI, after a median follow up of 8 years.[30] In the Optimal Trial in Myocardial Infarction with the Angiotensin II Antagonist Losartan (OPTIMAAL) trial, there was a strong positive association between moderate alcohol use and survival in patients with heart failure and/or evidence of left ventricular dysfunction following MI.[31] Persistent moderate alcohol drinking following an acute MI was associated with a trend toward less angina, fewer re-hospitalizations, lower 3-year mortality, and better disease-specific quality of life, and mental health than quitters.[32] In the Post-CABG trial, inverse trends were observed between moderate drinking and morbidity as well as graft disease progression.[33]

\section{Epidemiological Studies Showing Neutral Results of Alcohol on the Heart}


While a great many studies have shown favorable effects on CHD and all-cause mortality with light to moderate alcohol intake (Table 1), some studies have shown a neutral effect, and heavy alcohol consumption was shown to be detrimental. In the Framingham Study, cumulative incidence of alcohol use disorders did not show a decrease despite a decrease in average alcohol intake and more wine consumption after more than 50 years of followup.[34] Contrary to the cardioprotective effect of moderate regular alcohol consumption, heavy, episodic drinking was related to higher risk of $\mathrm{CHD},[35]$ and indeed in a prospective study of patients with MI, although moderate alcohol intake was associated with lower mortality, binge drinking, even among light drinkers, was associated with 2fold higher mortality.[36] A meta-analysis of 14 studies showed the cardioprotective effect of moderate alcohol consumption disappears when, on average, light to moderate drinking was mixed with irregular heavy drinking $(>60 \mathrm{~g}$ of pure alcohol or $>$ or $=5$ drinks per occasion at least monthly).[37] Former drinkers compared to long-term abstainers had significantly increased risk for CHD mortality, in a recent metaanalysis.[38] In a meta-analysis of six studies, a J-shaped curve, with nadir of approximately 28 grams of alcohol (2 drinks) per week, was observed including drinkers who consumed alcohol for 2 days a week or less. Conversely, in people who consumed alcohol for more than 2 days a week, a significant protective effect was seen even when drinking high amounts of alcohol.[39] In a meta-analysis of 28 cohort studies, there was a $20 \%$ relative risk reduction in CHD for up to $20 \mathrm{~g} /$ day of alcohol consumption, and evidence of a protective effect up to $72 \mathrm{~g} /$ day, but increased risk above $\geq 89 \mathrm{~g} /$ day.[40] 
The beneficial effects of light to moderate alcohol consumption on CHD could not be replicated in some studies, especially in studies of certain ethnic populations. Table 2 lists studies showing no favorable effects of light to moderate alcohol consumption on cardiovascular or all-cause mortality. In the NHANES Epidemiologic Follow-Up Study (NHEFS), no J-shaped beneficial relationship was found between alcohol consumption and mortality in African Americans. Conversely, mortality increased with increasing consumption of more than one drink a day in both genders.[41] Risk for all-cause mortality was similar for non-drinkers and men drinking up to 14 units per week ( 1 unit was equivalent to one measure of spirits, half a pint of beer, or one sixth bottle of wine) in Scottish men followed-up for 21 years, with an unfavorable outcome for drinking over 22 units a week.[42] In Asian-Indian men, no protective association was found between alcohol intake and prevalence of CHD. In fact, alcohol intake was associated with a higher odds ratio for CHD risk.[43, 44] Moderate-to-heavy alcohol consumption not only showed lack of benefit on CHD mortality in older Chinese men,[45] but also increased the risk of angiographically proven CAD.[46] In the Lipid Research Clinics Prevalence and Follow-up Studies, no difference in mortality rates were found between drinkers and non-drinkers in Russia.[47] In a cohort of males of high socioeconomic status, moderate alcohol consumption had no net benefit compared with abstinence, and $>3$ drinks per day was associated with worse quality of life, and adversely affected mortality after a 29-year follow-up.[48]

In a population based prospective study of 7169 men aged 45-64 years followed for a mean of 12.8 years, compared to occasional drinking, regular light alcohol consumption 
(1-14 units per week) in men with established CHD was not associated with any significant benefit, or all cause mortality. Higher levels of intake ( $>/=3$ drinks per day) were associated with increased mortality in men with previous MI.[49] In the MultiEthnic Study of Atherosclerosis (MESA) study, heavy consumption of hard liquor was associated with greater coronary artery calcium accumulation and there was no evidence of a protective or J-shaped association of alcohol and coronary artery calcium over a 2- to 4-y period.[50] Alcohol consumption was directly associated with carotid intima-media thickness in young healthy adults, independent of cardiovascular risk factors, suggesting a pro-atherogenic effect.[51]

Lack of replication of the well documented protective effect of moderate alcohol use in some studies could suggest that the protective effect of alcohol on CHD does not extend to all populations or that the confounding factors may not have been completely accounted for. Moreover, self reporting of alcohol consumption may have been less accurate rather than actual consumption. Other factors that may have contributed to the neutral effects of alcohol consumption include differences in the ethanol content, serving size, pattern of consumption, and phenotypic differences in the metabolism of alcohol. A meta-analysis of 54 all-cause mortality studies and 35 CHD mortality studies,[10] highlighted systemic,[52] and misclassification errors from the epidemiological studies, that demonstrated a J- or U-shaped benefit with alcohol consumption. However, this study was limited by the exclusion of some major studies which showed positive effects of alcohol on CHD. Another recent large systematic review and meta-analysis addressed 
this issue, and inclusion of former drinkers did not seem to bias the beneficial association of alcohol consumption with cardiovascular disease.[53]

\section{Alcohol Consumption and Vascular Disease}

Light to moderate alcohol consumption decreases risk of ischemic stroke, but heavy alcohol consumption may increase the risk of total stroke.[7, 54, 55] Moderate alcohol consumption also decreases risk of peripheral arterial disease.[56-59]

\section{Alcohol and Cardiovascular Risk Factors}

Moderate alcohol consumption has been associated with lower CHD risk in numerous, but not all, epidemiological studies. Alcohol reduces many but not all of the risk factors for CHD. The 2003 Behavioral Risk Factor Surveillance System data showed, while 27 of the $30 \mathrm{CHD}$-associated factors were significantly more prevalent in nondrinkers, compared to moderate drinkers, after adjusting for age and gender, few (if any) of these differences were likely due to alcohol consumption itself.[60] There was a J- or U-shaped relationship between alcohol intake and the prevalence of multiple cardiovascular risk factors, amongst non-smokers and smokers, in 27,005 middle aged Japanese men.[61] In the Italian Longitudinal Study on Aging (ILSA), moderate alcohol consumption at older age was associated with healthier hematological values of fibrinogen, HDL cholesterol (HDL-C), apo A-I lipoprotein and insulin. But it was also associated with a worse hematological picture of total cholesterol, LDL cholesterol (LDL-C) levels, and systolic blood pressure.[62]

\section{Alcohol and Lipids}


CHD risk reduction in light to moderate alcohol drinkers is generally attributed to a beneficial effect on HDL-C levels. However, this hypothesis was disputed recently in a large, population based Norwegian cohort (CONOR) study with extensive control for confounding factors.[63] Alcohol intake increases HDL-C in a dose-dependent fashion up to $18 \%$, associated with and possibly caused by an increase in the transportation rates of HDL apolipoproteins apoA-I and A-II.[64] HDL-C and apoA-I levels were significantly elevated in the Atherosclerosis Risk in Communities (ARIC) study, irrespective of the quantity or type of beverage consumed.[65] In a meta-analysis of 42 studies, $30 \mathrm{~g}$ of ethanol a day increased concentrations of HDL-C by approximately $4 \mathrm{mg} / \mathrm{dl}$, and apoA-I by $8.82 \mathrm{mg} / \mathrm{dl}$, with an estimated risk reduction of $24.7 \%$ in CHD.[66] In a recent systematic review and meta-analysis of 44 studies, alcohol significantly increased levels of HDL-C, apoA1, and adiponectin. Alcohol showed a dose-response relation with HDL-C, decreased fibrinogen levels, and did not affect triglyceride levels.[67]

Incremental alcohol intake was associated with significantly elevated levels of HDL-C, phospholipid enrichment of HDL, and a shift from HDL 3 subfraction to HDL 2, all of which, may augment the antiatherogenic effect of HDL-C.[68] Cholesterol efflux from macrophages to HDL (2) particles was significantly increased by heavy alcohol consumption, with associated decrease in cholesteryl ester transfer protein (CETP) activity, and increased phospholipid transfer protein (PLTP) activity.[69] Moderate alcohol consumption increased cholesterol efflux, possibly mediated by ATP-binding cassette transporter (ABCA1) in healthy men,[70] and in postmenopausal women.[71] 
Serum non-HDL cholesterol is a strong predictor of cardiovascular diseases. Even light drinking significantly lowered serum non-HDL cholesterol in healthy adults, with the effect more pronounced in women.[72] In the Cardiovascular Health Study, alcohol intake was associated with less total LDL particles, lower levels of small LDL, HDL, and very-low-density lipoprotein particles, and higher levels of large LDL and medium- and large-sized HDL particles, as measured by nuclear magnetic resonance (NMR) spectroscopy.[73]

Genetic polymorphisms and their interaction with alcohol consumption have been implicated in modulating serum lipid levels. Genetic variation in alcohol dehydrogenase 1C (ADH1C) and ADH3 have higher HDL levels and a substantially decreased risk of CHD in moderate drinkers, $[74,75]$ but this finding could not be replicated in the British Women's Heart and Health Study and Caerphilly cohorts.[76] Apolipoprotein E (APOE) polymorphism and its interaction with alcohol, altered LDL-C levels in the Framingham Offspring Study,[77] and in the Spanish EPIC cohort study.[78] Data from the Cohorte Lausannoise (CoLaus) study suggests that the effect of alcohol consumption on HDL-C levels may not be mediated by polymorphisms in APOA5, CETP, LIPC or LPL genes.[79]

\section{Alcohol and Diabetes Mellitus}

Light to moderate alcohol consumption was inversely associated with type 2 diabetes in 4,655 elderly people in the Cardiovascular Health Study,[80] 2,879 healthy adults in the 
Framingham Offspring Study,[81] and 20,951 physicians in the Physicians' Health Study after a median follow up of 12.1 years.[82] In a systematic review and meta-analysis comprising of 20 cohort studies, moderate alcohol consumption appeared protective for type 2 diabetes with a U-shaped relationship in both sexes.[83] Moderate alcohol consumption improved insulin sensitivity in relatively insulin-resistant middle-aged men, an effect that may be mediated through alcohol-induced increases in adiponectin.[84] In a nested case-control study, the inverse association between alcohol consumption and risk of type 2 diabetes was not explained by circulating biomarkers of inflammation, endothelial dysfunction, and fasting insulin, but adiponectin appeared to be a mediator.[85]

Light to moderate alcohol consumption was associated with lower risk of CHD in men with type 2 diabetes in the Health Professionals' Follow-up study (HPFS),[86] as well as in the Physicians' Health Study,[87] and decreased risk of death due to CHD.[88] In a meta-analysis of six cohort studies, moderate alcohol consumption was associated with a lower risk of total mortality and any alcohol consumption was associated with lower risk of CHD in type 2 diabetic populations.[89]

Alcohol consumption was also associated with a lower prevalence of metabolic syndrome in the NHLBI Family Heart Study, irrespective of the type of beverage consumed,[90] and in European population cohorts.[56, 91, 92] In a meta-analysis of 7 studies, alcohol consumption of less than $40 \mathrm{~g} / \mathrm{day}$ in men and $20 \mathrm{~g} /$ day in women significantly reduced the prevalence of metabolic syndrome.[93] 


\section{Alcohol and Hypertension}

Existing evidence is conflicting regarding the relation between alcohol consumption and systemic hypertension.

In a systematic review and meta-analysis comprising 12 cohort studies from the United States, Japan and Korea, the risk for hypertension increased linearly with alcohol consumption. Among women, a significant protective effect was reported for consumption at or below about $5 \mathrm{~g}$ per day. Among men, Asian populations had higher risks than non-Asian populations of hypertension associated with alcohol intake.[94] In the Coronary Artery Risk Development in Young Adults Study, 20-year follow up showed no association between alcohol consumption and incident hypertension, except among European-American women in whom any current alcohol consumption was associated with lower risk of incident hypertension.[95] In the ARIC study participants, the consumption of alcohol in amounts $\geq 210 \mathrm{~g}$ per week was an independent risk factor for hypertension in North America, after 6 years of follow-up. Light to moderate alcohol consumption also appeared to be associated with a higher risk of hypertension in black men.[96]

Light-to-moderate alcohol consumption decreased hypertension risk in women and increased risk in men, after prospective follow-ups of 10.9 and 21.8 years, in the Women's Health Study and Physicians' Health Study, respectively. The threshold above which alcohol became deleterious for hypertension risk emerged at $\geq 4$ drinks per day in women versus a moderate level of $\geq 1$ drink per day in men.[97] In Spanish men and 
women, consumption of beer or spirits, but not wine, was associated with a higher risk of developing hypertension, during a median follow-up of 4.2 years, irrespective of the pattern of consumption.[98]

The mechanisms that lead to alcohol-induced hypertension have not been well defined. In a study of healthy volunteers, higher blood pressure in drinkers, was not explained by increased activity of the sympatho-adrenal and renal-pressor mechanisms.[99] Light-tomoderate alcohol intake appeared to have a possible vasculoprotective effect, as measured by pulse wave velocity in subjects with optimal or normal blood pressure, while heavy drinking caused a detrimental effect on the vasculature.[100] Increased aortic pulse wave velocity was closely associated with alcohol consumption in middleaged Japanese men.[101]

Polymorphisms of alcohol-metabolizing enzymes (alcohol dehydrogenase (ADH) 2 and aldehyde dehydrogenase (ALDH) 2 may influence alcohol metabolism. One such polymorphism, $\mathrm{ADH} 2(1) / 2(1)$ genotype, was associated with lower levels of systolic blood pressure.[102] Genotypic variations in Catechol- O-methyltransferase (COMT) mRNA and the consequent genetically determined inactivation of catecholamines, may influence alcohol-induced blood pressure elevation.[103]

\section{Alcohol and Coagulation}

Epidemiological studies indicate that consumption of alcohol at the average level of intake in France (20-30 g per day) reduced risk of CHD by at least 40\%, despite a high 
intake of saturated fat. This "French Paradox," may be attributable to regular wine consumption. Alcohol is believed to protect from CHD by preventing atherosclerosis through action on HDL-C, but serum concentrations were no higher in France than in other countries. The effect of wine in lowering the platelet reactivity, as seen in France compared to Scotland, may be one explanation for this protection.[104]

In the Framingham Offspring Study, light-to-moderate alcohol consumption was associated with lower levels of coagulatory factors (fibrinogen, plasma viscosity, von Willebrand factor, and factor VII), but higher intake was associated with impaired fibrinolytic potential, reflected by higher levels of plasminogen activator inhibitor antigen-1 and tissue plasminogen activator antigen. These findings are consistent with the hypothesis that a balance between hemostatic and fibrinolytic activity may contribute to the complex relation of alcohol use with coronary heart disease.[105, 106]

A dose-dependent effect of alcohol on decreased platelet aggregation was also seen in the Caerphilly Prospective Heart Disease Study.[107] In a meta-analysis of 42 studies, $30 \mathrm{~g}$ of daily alcohol consumption modestly increased concentrations of several haemostatic factors including fibrinogen.[66] Wine drinkers had lower levels of plasminogen activator inhibitor (PAI) antigen-1, and ADP-mediated platelet activation and aggregation compared to others.[66]

Moderate alcohol intake was positively associated with plasma level of endogenous t-PA, independent of HDL-C in the Physicians' Health Study.[108] In the NHLBI Family Heart 
Study, alcohol consumption > 15g/d was associated with increased PAI-1.[109] These findings support the hypothesis that changes in fibrinolytic potential may be an important mechanism whereby moderate alcohol consumption decreases risk of heart disease. Moderate alcohol consumption also improved endothelial function as evidenced by better flow-mediated dilation (FMD) in the Northern Manhattan Study (NOMAS).[110]

\section{Alcohol and Inflammation}

In view of the robust association between markers of inflammation, especially C-reactive protein (CRP), and risk of CHD, an anti-inflammatory action of alcohol could contribute to the link between moderate consumption and lower cardiovascular mortality. Multiple studies have demonstrated the beneficial effects of moderate alcohol consumption on various inflammatory markers with significantly decreased levels of plasma CRP, fibrinogen, interleukin-6 (IL-6), interleukin-1alpha, as well as plasma viscosity, WBC count, factor VIIIc, and monocyte and endothelial adhesion molecules.[111-115] Men who consumed 1-2 drinks/day had 26\% lower CRP, and 36\% lower IL-6 levels, with a stronger association in women.[116] Lower CRP concentrations were also observed in the Pravastatin Inflammation/CRP Evaluation Study, independent of alcohol-related effects on lipids.[117] These results suggest an additional biological explanation to the epidemiological link between moderate alcohol consumption and decreased cardiovascular events.

\section{Alcohol Preconditioning}

Epidemiological data suggest that those who are regularly consuming alcohol prior to an acute MI are more likely to survive, suggesting a preconditioning effect of alcohol.[28, 
118] Ischemic preconditioning (IPC) is the most potent form of endogenous myocardial protection against irreversible ischemia-reperfusion injury. In an animal models, longterm alcohol consumption reduced myocardial ischemia-reperfusion injury possibly through repeated myocyte adenosine receptor activation,[119] and sustained translocation of epsilon protein kinase $\mathrm{C}(\varepsilon \mathrm{PKC})$.[120] In situ studies in rabbit showed, ethanol exposure followed by washout or sufficient time to metabolize the alcohol prior to ischemia, induces preconditioning-like myocardial protection.[121] Protection afforded against ischemia and reperfusion injury (I/R) proceeds through an ischemic preconditioning-like mechanism involving the activation of $\varepsilon \mathrm{PKC}$ that is dependent on the time and duration of ethanol treatment.[122, 123]

Of note, moderate dose of ethanol when taken acutely, abolished ischemic preconditioning (IPC) occurring during sequential episodes of myocardial ischemia, and is associated with worsening ischemia in a randomized prospective study in humans.[124]

\section{Wine versus other Alcoholic Beverages}

Some but not all, epidemiological studies suggest that wine confers greater protection against CHD than beer or distilled spirits, by virtue of its polyphenol content.[125] In a systematic review and meta-analysis, small doses (1-4 drinks/day) of wine, beer, and spirits were equally beneficial in reducing CHD.[126] Another systematic review that included ecological, case-control, and prospective cohort studies, also showed that all types of alcoholic drinks were linked with lower risk of CHD.[127] However, in a metaanalysis, relative risk of vascular disease associated with wine intake was 0.68 (95\% confidence interval, 0.59 to 0.77 ) in 209418 persons compared to non-drinkers, with a strong J-shaped relationship between different amounts of wine intake and vascular risk. 
A similar but smaller association was also apparent with beer consumption, but no meaningful relationship could be found between different amounts of beer intake and vascular risk.[128] Potential protective mechanisms beyond the effects of ethanol include, greater anti-oxidant effects,[129-131] decreased erythrocyte superoxide dismutase activity,[132] and in vitro studies showing reduced vascular smooth muscle cells proliferation,[133] vasorelaxation of human coronary arteries,[134] inhibition of endothelin-1 synthesis,[135] and down-regulation of tissue factor (TF) gene transcription.[136]

\section{Alcohol and Heart Failure}

Moderate alcohol consumption was associated with a lower risk of incident heart failure among older adults and in the Physicians' Health Study, independent of a number of confounding factors. Further, it did not appear to be mediated by a reduction in MI risk, after an extended follow-up of 18 years.[137-139] Light to moderate drinking was associated with a lower risk of HF in hypertensive male physicians.[140] In the Framingham Heart Study, during 26,035 person-years of follow-up in men, and 35,563 person-years of follow-up in women, alcohol consumption was not associated with increased risk for heart failure, even among heavy drinkers $(\geq 15$ drinks/wk in men and $\geq$ 8 drinks/wk in women). To the contrary, moderate alcohol consumption protected against heart failure.[141] In a meta-analysis of 6 studies, infrequent and light-to-moderate drinking was associated with a $10 \%$ to $20 \%$ lower risk of heart failure.[142] Heavy alcohol drinking was associated with increased risk of non-ischemic cardiomyopathy, and 
the apparent protection of alcohol against ischemic cardiomyopathy, supported its protective effect against CHD.[143]

Alcohol is toxic to striated muscle in a dose-dependent manner, and in patients with alcoholic cardiomyopathy, total lifetime dose of ethanol correlated inversely with the ejection fraction and directly with the left ventricular mass.[144] In the Framingham Offspring Study, alcohol intake was positively and independently associated with left ventricular mass in men but not in women.[145] Subtle signs of cardiac abnormalities such as left ventricular dilation with preserved ejection fraction and impaired left ventricular relaxation may precede severe cardiac dysfunction in chronic asymptomatic alcoholics.[146] Changes in myocardial contractility due to chronic alcohol exposure do not result from altered $\mathrm{Ca}^{2+}$ handling but from changes at the level of the myofilament that do not involve myosin heavy chain isoform shifts.[147] Transgenic overexpression of insulinlike growth factor 1 attenuated the deleterious effects caused by chronic alcohol intake such as interstitial fibrosis, reduced mitochondrial number, compromised cardiac contractile function and intracellular $\mathrm{Ca}(2+)$ handling, decreased superoxide dismutase (SOD) 1 expression, elevated superoxide production, and overt apoptosis, with the exception of cardiac hypertrophy.[148]

Abstinence is the most critical step in halting further deterioration of cardiac contractility in alcoholics. In a 4-year prospective cohort study in Spain, left ventricular ejection fraction improved in both abstinent patients and those who controlled their drinking up to 60 grams of ethanol per day.[149] 
Like the relationship between alcohol and CHD, the relationship between alcohol and CHF is complex. While light to moderate alcohol intake may be protective, heavy consumption increases heart failure risk.

\section{Alcohol and Atrial Fibrillation}

Moderate alcohol consumption was not significantly associated with a risk of atrial fibrillation, $[150,151]$ but the risk significantly increased when alcohol consumption was $>36 \mathrm{~g}$ /day during a follow up of > 50 years in the Framingham Study.[150] Heavy alcohol consumption was associated with atrial fibrillation in 5\% of cases in the Copenhagen City Heart Study.[152] In a recent meta-analysis of 14 studies, a linear relationship was found between atrial fibrillation risk and alcohol consumption, suggesting abstinence from alcohol is most favorable in terms of atrial fibrillation risk reduction.[153]

\section{Conclusion}

Current guidelines recommend limiting alcohol intake to one drink per day for women, and two drinks per day for men.[154] Existing epidemiological data clearly demonstrates many favorable biological effects (Figure 3) of light to moderate alcohol consumption on the cardiovascular system. These include cardioprotective, anti-inflammatory, anti-oxidant, and hemorheological effects, as well as modulation of cardiovascular risk factors. A large body of epidemiological data supports the relationship between light to moderate alcohol consumption and a reduction in cardiovascular and all-cause mortality; predominantly seen in western populations. However, a small number of studies did not demonstrate a benefit of light to moderate alcohol consumption, especially in certain ethnic populations. This may 
partly be explained by genetic and metabolic factors, but questions remain regarding self-reported data collection in some studies and other confounding factors that may not have been accounted for. Further robust studies are needed in certain ethnic populations where these benefits could not be replicated.

Cardiovascular benefits of low to moderate alcohol consumption give way to cardiovascular complications with heavy or binge drinking. These can include cardiomyopathy and heart failure, hypertension, arrhythmias, and stroke. Routine recommendations to initiate alcohol consumption to prevent cardiovascular disease should not be made in the absence of data from randomized controlled trials and the risk of abuse and addiction. However, in patients at risk for cardiovascular events who already responsibly consume alcohol, there is little reason to encourage abstinence. 
1. Rehm, J., et al., Global burden of disease and injury and economic cost attributable to alcohol use and alcohol-use disorders. Lancet, 2009. 373(9682): p. 2223-33.

2. Li, T.K., B.G. Hewitt, and B.F. Grant, The Alcohol Dependence Syndrome, 30 years later: a commentary. the $2006 \mathrm{H}$. David Archibald lecture. Addiction, 2007. 102(10): p. 1522-30.

3. Hasin, D.S., et al., Prevalence, correlates, disability, and comorbidity of DSMIV alcohol abuse and dependence in the United States: results from the National Epidemiologic Survey on Alcohol and Related Conditions. Arch Gen Psychiatry, 2007. 64(7): p. 830-42.

4. Alcohol-attributable deaths and years of potential life lost--United States, 2001. MMWR Morb Mortal Wkly Rep, 2004. 53(37): p. 866-70.

5. Mokdad, A.H., et al., Actual causes of death in the United States, 2000. JAMA, 2004. 291(10): p. 1238-45.

6. Klatsky, A.L., G.D. Friedman, and A.B. Siegelaub, Alcohol and mortality. A ten-year Kaiser-Permanente experience. Ann Intern Med, 1981. 95(2): p. 13945.

7. Stampfer, M.J., et al., A prospective study of moderate alcohol consumption and the risk of coronary disease and stroke in women. N Engl J Med, 1988. 319(5): p. 267-73.

8. Di Castelnuovo, A., et al., Alcohol dosing and total mortality in men and women: an updated meta-analysis of 34 prospective studies. Arch Intern Med, 2006. 166(22): p. 2437-45.

9. Costanzo, S., et al., Alcohol consumption and mortality in patients with cardiovascular disease: a meta-analysis. J Am Coll Cardiol, 2010. 55(13): p. 1339-47.

10. Fillmore, K.M., et al., Moderate alcohol use and reduced mortality risk: systematic error in prospective studies and new hypotheses. Ann Epidemiol, 2007. 17(5 Suppl): p. S16-23.

11. Friedman, L.A. and A.W. Kimball, Coronary heart disease mortality and alcohol consumption in Framingham. Am J Epidemiol, 1986. 124(3): p. 481-9.

12. Mukamal, K.J., et al., Alcohol consumption and cardiovascular mortality among U.S. adults, 1987 to 2002. J Am Coll Cardiol, 2010. 55(13): p. 1328-35.

13. Hvidtfeldt, U.A., et al., Alcohol intake and risk of coronary heart disease in younger, middle-aged, and older adults. Circulation, 2010. 121(14): p. 1589-97.

14. Mukamal, K.J., S.E. Chiuve, and E.B. Rimm, Alcohol consumption and risk for coronary heart disease in men with healthy lifestyles. Arch Intern Med, 2006. 166(19): p. 2145-50.

15. Bazzano, L.A., et al., Alcohol consumption and risk of coronary heart disease among Chinese men. Int J Cardiol, 2009. 135(1): p. 78-85.

16. Inoue, M., et al., Impact of alcohol intake on total mortality and mortality from major causes in Japan: a pooled analysis of six large-scale cohort studies. J Epidemiol Community Health, 2010. 
17. Djousse, L., et al., Alcohol consumption and risk of cardiovascular disease and death in women: potential mediating mechanisms. Circulation, 2009. 120(3): p. 237-44.

18. Ikehara, S., et al., Alcohol consumption and mortality from stroke and coronary heart disease among Japanese men and women: the Japan collaborative cohort study. Stroke, 2008. 39(11): p. 2936-42.

19. Freiberg, M.S., et al., Alcohol consumption, hypertension, and total mortality among women. Am J Hypertens, 2009. 22(11): p. 1212-8.

20. Behrens, G., et al., The association between alcohol consumption and mortality: the Swedish women's lifestyle and health study. Eur J Epidemiol, 2011. 26(2): p. 81-90.

21. Rajpathak, S.N., et al., Alcohol consumption and the risk of coronary heart disease in postmenopausal women with diabetes: Women's Health Initiative Observational Study. Eur J Nutr, 2010. 49(4): p. 211-8.

22. Solomon, C.G., et al., Moderate alcohol consumption and risk of coronary heart disease among women with type 2 diabetes mellitus. Circulation, 2000. 102(5): p. 494-9.

23. Janszky, I., et al., Alcohol consumption and coronary atherosclerosis progression--the Stockholm Female Coronary Risk Angiographic Study. Atherosclerosis, 2004. 176(2): p. 311-9.

24. Beulens, J.W., et al., Alcohol consumption and risk of recurrent cardiovascular events and mortality in patients with clinically manifest vascular disease and diabetes mellitus: the Second Manifestations of ARTerial (SMART) disease study. Atherosclerosis, 2010. 212(1): p. 281-6.

25. Ruidavets, J.B., et al., Patterns of alcohol consumption and ischaemic heart disease in culturally divergent countries: the Prospective Epidemiological Study of Myocardial Infarction (PRIME). BMJ, 2010. 341: p. c6077.

26. Britton, K.A., et al., Relation of alcohol consumption and coronary heart disease in hypertensive male physicians (from the Physicians' Health Study). Am J Cardiol, 2009. 104(7): p. 932-5.

27. Beulens, J.W., et al., Alcohol consumption and risk for coronary heart disease among men with hypertension. Ann Intern Med, 2007. 146(1): p. 10-9.

28. Mukamal, K.J., et al., Prior alcohol consumption and mortality following acute myocardial infarction. JAMA, 2001. 285(15): p. 1965-70.

29. de Lorgeril, M., et al., Wine drinking and risks of cardiovascular complications after recent acute myocardial infarction. Circulation, $2002.106(12):$ p. 1465-9.

30. Janszky, I., et al., Alcohol and long-term prognosis after a first acute myocardial infarction: the SHEEP study. Eur Heart J, 2008. 29(1): p. 45-53.

31. Brugger-Andersen, T., et al., Moderate alcohol consumption is associated with reduced long-term cardiovascular risk in patients following a complicated acute myocardial infarction. Int J Cardiol, 2009. 133(2): p. 229-32.

32. Carter, M.D., et al., Comparison of outcomes among moderate alcohol drinkers before acute myocardial infarction to effect of continued versus discontinuing alcohol intake after the infarct. Am J Cardiol, 2010. 105(12): p. 1651-4. 
33. Mukamal, K.J., S. Girotra, and M.A. Mittleman, Alcohol consumption, atherosclerotic progression, and prognosis among patients with coronary artery bypass grafts. Am Heart J, 2006. 151(2): p. 368-72.

34. Zhang, Y., et al., Secular trends in alcohol consumption over 50 years: the Framingham Study. Am J Med, 2008. 121(8): p. 695-701.

35. McElduff, P. and A.J. Dobson, How much alcohol and how often? Population based case-control study of alcohol consumption and risk of a major coronary event. BMJ, 1997. 314(7088): p. 1159-64.

36. Mukamal, K.J., et al., Binge drinking and mortality after acute myocardial infarction. Circulation, 2005. 112(25): p. 3839-45.

37. Roerecke, M. and J. Rehm, Irregular heavy drinking occasions and risk of ischemic heart disease: a systematic review and meta-analysis. Am J Epidemiol, 2010. 171(6): p. 633-44.

38. Roerecke, M. and J. Rehm, Ischemic heart disease mortality and morbidity rates in former drinkers: a meta-analysis. Am J Epidemiol, 2011. 173(3): p. 245-58.

39. Bagnardi, V., et al., Does drinking pattern modify the effect of alcohol on the risk of coronary heart disease? Evidence from a meta-analysis. J Epidemiol Community Health, 2008. 62(7): p. 615-9.

40. Corrao, G., et al., Alcohol and coronary heart disease: a meta-analysis. Addiction, 2000. 95(10): p. 1505-23.

41. Sempos, C.T., et al., Average volume of alcohol consumption and all-cause mortality in African Americans: the NHEFS cohort. Alcohol Clin Exp Res, 2003. 27(1): p. 88-92.

42. Hart, C.L., et al., Alcohol consumption and mortality from all causes, coronary heart disease, and stroke: results from a prospective cohort study of scottish men with 21 years of follow up. BMJ, 1999. 318(7200): p. 1725-9.

43. Roy, A., et al., Impact of alcohol on coronary heart disease in Indian men. Atherosclerosis, 2010. 210(2): p. 531-5.

44. Joshi, P., et al., Risk factors for early myocardial infarction in South Asians compared with individuals in other countries. JAMA, 2007. 297(3): p. 286-94.

45. Schooling, C.M., et al., Moderate alcohol use and mortality from ischaemic heart disease: a prospective study in older Chinese people. PLoS One, 2008. 3(6): p. e2370.

46. Zhou, $X$., et al., Relation of alcohol consumption to angiographically proved coronary artery disease in chinese men. Am J Cardiol, 2010. 106(8): p. 1101-3.

47. Deev, A., et al., Association of alcohol consumption to morality in middle-aged U.S. and Russian men and women. Ann Epidemiol, 1998. 8(3): p. 147-53.

48. Strandberg, A.Y., et al., Alcohol consumption, 29-y total mortality, and quality of life in men in old age. Am J Clin Nutr, 2004. 80(5): p. 1366-71.

49. Shaper, A.G. and S.G. Wannamethee, Alcohol intake and mortality in middle aged men with diagnosed coronary heart disease. Heart, 2000. 83(4): p. 394-9.

50. McClelland, R.L., et al., Alcohol and coronary artery calcium prevalence, incidence, and progression: results from the Multi-Ethnic Study of

Atherosclerosis (MESA). Am J Clin Nutr, 2008. 88(6): p. 1593-601. 
51. Juonala, M., et al., Alcohol consumption is directly associated with carotid intima-media thickness in Finnish young adults: the Cardiovascular Risk in Young Finns Study. Atherosclerosis, 2009. 204(2): p. e93-8.

52. Shaper, A.G., G. Wannamethee, and M. Walker, Alcohol and mortality in British men: explaining the U-shaped curve. Lancet, 1988. 2(8623): p. 1267-73.

53. Ronksley, P.E., et al., Association of alcohol consumption with selected cardiovascular disease outcomes: a systematic review and meta-analysis. BMJ, 2011. 342: p. d671.

54. Reynolds, K., et al., Alcohol consumption and risk of stroke: a meta-analysis. JAMA, 2003. 289(5): p. 579-88.

55. Berger, K., et al., Light-to-moderate alcohol consumption and risk of stroke among U.S. male physicians. N Engl J Med, 1999. 341(21): p. 1557-64.

56. Athyros, V.G., et al., Association of drinking pattern and alcohol beverage type with the prevalence of metabolic syndrome, diabetes, coronary heart disease, stroke, and peripheral arterial disease in a Mediterranean cohort. Angiology, 2007. 58(6): p. 689-97.

57. Vliegenthart, R., et al., Alcohol consumption and risk of peripheral arterial disease: the Rotterdam study. Am J Epidemiol, 2002. 155(4): p. 332-8.

58. Camargo, C.A., Jr., et al., Prospective study of moderate alcohol consumption and risk of peripheral arterial disease in US male physicians. Circulation, 1997. 95(3): p. 577-80.

59. Jepson, R.G., et al., Alcohol intake as a risk factor for peripheral arterial disease in the general population in the Edinburgh Artery Study. Eur J Epidemiol, 1995. 11(1): p. 9-14.

60. Naimi, T.S., et al., Cardiovascular risk factors and confounders among nondrinking and moderate-drinking U.S. adults. Am J Prev Med, 2005. 28(4): p. 369-73.

61. Wakabayashi, I., Associations between alcohol drinking and multiple risk factors for atherosclerosis in smokers and nonsmokers. Angiology, 2010. 61(5): p. 495-503.

62. Perissinotto, E., et al., Alcohol consumption and cardiovascular risk factors in older lifelong wine drinkers: the Italian Longitudinal Study on Aging. Nutr Metab Cardiovasc Dis, 2010. 20(9): p. 647-55.

63. Magnus, P., et al., Controlling for high-density lipoprotein cholesterol does not affect the magnitude of the relationship between alcohol and coronary heart disease. Circulation, 2011. 124(21): p. 2296-302.

64. De Oliveira, E.S.E.R., et al., Alcohol consumption raises HDL cholesterol levels by increasing the transport rate of apolipoproteins $A-I$ and $A-I I$. Circulation, 2000. 102(19): p. 2347-52.

65. Volcik, K.A., et al., Relationship of alcohol consumption and type of alcoholic beverage consumed with plasma lipid levels: differences between Whites and African Americans of the ARIC study. Ann Epidemiol, 2008. 18(2): p. 101-7.

66. Rimm, E.B., et al., Moderate alcohol intake and lower risk of coronary heart disease: meta-analysis of effects on lipids and haemostatic factors. BMJ, 1999. 319(7224): p. 1523-8. 
67. Brien, S.E., et al., Effect of alcohol consumption on biological markers associated with risk of coronary heart disease: systematic review and metaanalysis of interventional studies. BMJ, 2011. 342: p. d636.

68. Schafer, C., et al., Beyond HDL-cholesterol increase: phospholipid enrichment and shift from HDL3 to HDL2 in alcohol consumers. J Lipid Res, 2007. 48(7): p. 1550-8.

69. Makela, S.M., et al., HDL2 of heavy alcohol drinkers enhances cholesterol efflux from raw macrophages via phospholipid-rich $H D L 2 b$ particles. Alcohol Clin Exp Res, 2008. 32(6): p. 991-1000.

70. Beulens, J.W., et al., Moderate alcohol consumption increases cholesterol efflux mediated by ABCA1. J Lipid Res, 2004. 45(9): p. 1716-23.

71. Sierksma, A., et al., Effect of moderate alcohol consumption on parameters of reverse cholesterol transport in postmenopausal women. Alcohol Clin Exp Res, 2004. 28(4): p. 662-6.

72. Wakabayashi, I. and K. Groschner, Modification of the association between alcohol drinking and non-HDL cholesterol by gender. Clin Chim Acta, 2009. 404(2): p. 154-9.

73. Mukamal, K.J., et al., Alcohol consumption and lipoprotein subclasses in older adults. J Clin Endocrinol Metab, 2007. 92(7): p. 2559-66.

74. Hines, L.M., et al., Genetic variation in alcohol dehydrogenase and the beneficial effect of moderate alcohol consumption on myocardial infarction. $\mathbf{N}$ Engl J Med, 2001. 344(8): p. 549-55.

75. Younis, J., et al., Genetic variation in alcohol dehydrogenase $1 C$ and the beneficial effect of alcohol intake on coronary heart disease risk in the Second Northwick Park Heart Study. Atherosclerosis, 2005. 180(2): p. 225-32.

76. Ebrahim, S., et al., Alcohol dehydrogenase type $1 C$ (ADH1C) variants, alcohol consumption traits, HDL-cholesterol and risk of coronary heart disease in women and men: British Women's Heart and Health Study and Caerphilly cohorts. Atherosclerosis, 2008. 196(2): p. 871-8.

77. Corella, D., et al., Alcohol drinking determines the effect of the APOE locus on LDL-cholesterol concentrations in men: the Framingham Offspring Study. Am J Clin Nutr, 2001. 73(4): p. 736-45.

78. Corella, D., et al., Saturated fat intake and alcohol consumption modulate the association between the APOE polymorphism and risk of future coronary heart disease: a nested case-control study in the Spanish EPIC cohort. J Nutr Biochem, 2010.

79. Marques-Vidal, P., et al., No interaction between alcohol consumption and HDL-related genes on HDL cholesterol levels. Atherosclerosis, 2010. 211(2): p. 551-7.

80. Djousse, L., et al., Alcohol consumption and type 2 diabetes among older adults: the Cardiovascular Health Study. Obesity (Silver Spring), 2007. 15(7): p. 1758-65.

81. Imamura, F., et al., Confounding by dietary patterns of the inverse association between alcohol consumption and type 2 diabetes risk. Am J Epidemiol, 2009. 170(1): p. 37-45. 
82. Ajani, U.A., et al., Alcohol consumption and risk of type 2 diabetes mellitus among US male physicians. Arch Intern Med, 2000. 160(7): p. 1025-30.

83. Baliunas, D.O., et al., Alcohol as a risk factor for type 2 diabetes: A systematic review and meta-analysis. Diabetes Care, 2009. 32(11): p. 2123-32.

84. Sierksma, A., et al., Effect of moderate alcohol consumption on adiponectin, tumor necrosis factor-alpha, and insulin sensitivity. Diabetes Care, 2004. 27(1): p. 184-9.

85. Beulens, J.W., et al., Alcohol consumption, mediating biomarkers, and risk of type 2 diabetes among middle-aged women. Diabetes Care, 2008. 31(10): p. 2050-5.

86. Tanasescu, M., et al., Alcohol consumption and risk of coronary heart disease among men with type 2 diabetes mellitus. J Am Coll Cardiol, 2001. 38(7): p. 1836-42.

87. Ajani, U.A., et al., Alcohol consumption and risk of coronary heart disease by diabetes status. Circulation, 2000. 102(5): p. 500-5.

88. Valmadrid, C.T., et al., Alcohol intake and the risk of coronary heart disease mortality in persons with older-onset diabetes mellitus. JAMA, 1999. 282(3): p. 239-46.

89. Koppes, L.L., et al., Meta-analysis of the relationship between alcohol consumption and coronary heart disease and mortality in type 2 diabetic patients. Diabetologia, 2006. 49(4): p. 648-52.

90. Djousse, L., et al., Alcohol consumption and metabolic syndrome: does the type of beverage matter? Obes Res, 2004. 12(9): p. 1375-85.

91. Husemoen, L.L., et al., The association of alcohol and alcohol metabolizing gene variants with diabetes and coronary heart disease risk factors in a white population. PLoS One, 2010. 5(8): p.e11735.

92. Buja, A., et al., Alcohol consumption and metabolic syndrome in the elderly: results from the Italian longitudinal study on aging. Eur J Clin Nutr, 2010. 64(3): p. 297-307.

93. Alkerwi, A., et al., Alcohol consumption and the prevalence of metabolic syndrome: a meta-analysis of observational studies. Atherosclerosis, 2009. 204(2): p. 624-35.

94. Taylor, B., et al., Alcohol and hypertension: gender differences in doseresponse relationships determined through systematic review and metaanalysis. Addiction, 2009. 104(12): p. 1981-90.

95. Halanych, J.H., et al., Alcohol consumption in young adults and incident hypertension: 20-year follow-up from the Coronary Artery Risk Development in Young Adults Study. Am J Epidemiol, 2010. 171(5): p. 532-9.

96. Fuchs, F.D., et al., Alcohol consumption and the incidence of hypertension: The Atherosclerosis Risk in Communities Study. Hypertension, 2001. 37(5): p. 1242-50.

97. Sesso, H.D., et al., Alcohol consumption and the risk of hypertension in women and men. Hypertension, 2008. 51(4): p. 1080-7.

98. Nunez-Cordoba, J.M., et al., Alcohol consumption and the incidence of hypertension in a Mediterranean cohort: the SUN study. Rev Esp Cardiol, 2009. 62(6): p. 633-41. 
99. Arkwright, P.D., et al., The pressor effect of moderate alcohol consumption in man: a search for mechanisms. Circulation, 1982. 66(3): p. 515-9.

100. Matsumoto, C., et al., Association of blood pressure levels with the effects of alcohol intake on the vasculature in Japanese men. Hypertens Res, 2009. 32(2): p. 127-32.

101. Nakanishi, N., et al., Alcohol consumption and risk for increased aortic pulse wave velocity in middle-aged Japanese men. Angiology, 2001. 52(8): p. 533-42.

102. Hashimoto, Y., et al., Relationship between genetic polymorphisms of alcoholmetabolizing enzymes and changes in risk factors for coronary heart disease associated with alcohol consumption. Clin Chem, 2002. 48(7): p. 1043-8.

103. Stewart, S.H., et al., COMT genotype influences the effect of alcohol on blood pressure: results from the COMBINE study. Am J Hypertens, 2009. 22(1): p. 87-91.

104. Renaud, S. and M. de Lorgeril, Wine, alcohol, platelets, and the French paradox for coronary heart disease. Lancet, 1992. 339(8808): p. 1523-6.

105. Mukamal, K.J., et al., Alcohol consumption and hemostatic factors: analysis of the Framingham Offspring cohort. Circulation, 2001. 104(12): p. 1367-73.

106. Mukamal, K.J., et al., Alcohol consumption and platelet activation and aggregation among women and men: the Framingham Offspring Study. Alcohol Clin Exp Res, 2005. 29(10): p. 1906-12.

107. Renaud, S.C., et al., Alcohol and platelet aggregation: the Caerphilly Prospective Heart Disease Study. Am J Clin Nutr, 1992. 55(5): p. 1012-7.

108. Ridker, P.M., et al., Association of moderate alcohol consumption and plasma concentration of endogenous tissue-type plasminogen activator. JAMA, 1994. 272(12): p. 929-33.

109. Djousse, L., et al., Alcohol consumption and plasminogen activator inhibitor type 1: the National Heart, Lung, and Blood Institute Family Heart Study. Am Heart J, 2000. 139(4): p. 704-9.

110. Suzuki, K., et al., Moderate alcohol consumption is associated with better endothelial function: a cross sectional study. BMC Cardiovasc Disord, 2009. 9: p. 8.

111. Sierksma, A., et al., Moderate alcohol consumption reduces plasma C-reactive protein and fibrinogen levels; a randomized, diet-controlled intervention study. Eur J Clin Nutr, 2002. 56(11): p. 1130-6.

112. Imhof, A., et al., Overall alcohol intake, beer, wine, and systemic markers of inflammation in western Europe: results from three MONICA samples (Augsburg, Glasgow, Lille). Eur Heart J, 2004. 25(23): p. 2092-100.

113. Volpato, S., et al., Relationship of alcohol intake with inflammatory markers and plasminogen activator inhibitor-1 in well-functioning older adults: the Health, Aging, and Body Composition study. Circulation, 2004. 109(5): p. 60712.

114. Estruch, R., et al., Different effects of red wine and gin consumption on inflammatory biomarkers of atherosclerosis: a prospective randomized crossover trial. Effects of wine on inflammatory markers. Atherosclerosis, 2004. 175(1): p. 117-23. 
115. Mukamal, K.J., et al., Alcohol consumption and inflammatory markers in older adults: the Cardiovascular Health Study. Atherosclerosis, 2004. 173(1): p. 79-87.

116. Pai, J.K., et al., Moderate alcohol consumption and lower levels of inflammatory markers in US men and women. Atherosclerosis, 2006. 186(1): p. 113-20.

117. Albert, M.A., R.J. Glynn, and P.M. Ridker, Alcohol consumption and plasma concentration of $C$-reactive protein. Circulation, 2003. 107(3): p. 443-7.

118. Wannamethee, G., et al., Factors determining case fatality in myocardial infarction "who dies in a heart attack"? Br Heart J, 1995. 74(3): p. 324-31.

119. Miyamae, M., et al., Regular alcohol consumption mimics cardiac preconditioning by protecting against ischemia-reperfusion injury. Proc Natl Acad Sci U S A, 1997. 94(7): p. 3235-9.

120. Miyamae, M., et al., Activation of epsilon protein kinase C correlates with a cardioprotective effect of regular ethanol consumption. Proc Natl Acad Sci U S A, 1998. 95(14): p. 8262-7.

121. Krenz, M., et al., Acute ethanol exposure fails to elicit preconditioning-like protection in in situ rabbit hearts because of its continued presence during ischemia. J Am Coll Cardiol, 2001. 37(2): p. 601-7.

122. Churchill, E.N., M.H. Disatnik, and D. Mochly-Rosen, Time-dependent and ethanol-induced cardiac protection from ischemia mediated by mitochondrial translocation of varepsilonPKC and activation of aldehyde dehydrogenase 2 . J Mol Cell Cardiol, 2009. 46(2): p. 278-84.

123. Zhou, H.Z., J.S. Karliner, and M.O. Gray, Moderate alcohol consumption induces sustained cardiac protection by activating PKC-epsilon and Akt. Am J Physiol Heart Circ Physiol, 2002. 283(1): p. H165-74.

124. Niccoli, G., et al., Ethanol abolishes ischemic preconditioning in humans. J Am Coll Cardiol, 2008. 51(3): p. 271-5.

125. Streppel, M.T., et al., Long-term wine consumption is related to cardiovascular mortality and life expectancy independently of moderate alcohol intake: the Zutphen Study. J Epidemiol Community Health, 2009. 63(7): p. 534-40.

126. Cleophas, T.J., Wine, beer and spirits and the risk of myocardial infarction: a systematic review. Biomed Pharmacother, 1999. 53(9): p. 417-23.

127. Rimm, E.B., et al., Review of moderate alcohol consumption and reduced risk of coronary heart disease: is the effect due to beer, wine, or spirits. BMJ, 1996. 312(7033): p. 731-6.

128. Di Castelnuovo, A., et al., Meta-analysis of wine and beer consumption in relation to vascular risk. Circulation, 2002. 105(24): p. 2836-44.

129. Aviram, M. and B. Fuhrman, Wine flavonoids protect against LDL oxidation and atherosclerosis. Ann N Y Acad Sci, 2002. 957: p. 146-61.

130. Pignatelli, P., et al., Polyphenols synergistically inhibit oxidative stress in subjects given red and white wine. Atherosclerosis, 2006. 188(1): p. 77-83.

131. Covas, M.I., et al., Wine and oxidative stress: up-to-date evidence of the effects of moderate wine consumption on oxidative damage in humans.

Atherosclerosis, 2010. 208(2): p. 297-304. 
132. Estruch, R., et al., Moderate consumption of red wine, but not gin, decreases erythrocyte superoxide dismutase activity: a randomised cross-over trial. Nutr Metab Cardiovasc Dis, 2011. 21(1): p. 46-53.

133. Araim, O., et al., Inhibition of vascular smooth muscle cell proliferation with red wine and red wine polyphenols. J Vasc Surg, 2002. 35(6): p. 1226-32.

134. Flesch, M., A. Schwarz, and M. Bohm, Effects of red and white wine on endothelium-dependent vasorelaxation of rat aorta and human coronary arteries. Am J Physiol, 1998. 275(4 Pt 2): p. H1183-90.

135. Corder, R., et al., Endothelin-1 synthesis reduced by red wine. Nature, 2001. 414(6866): p. 863-4.

136. Casani, L., et al., Moderate daily intake of red wine inhibits mural thrombosis and monocyte tissue factor expression in an experimental porcine model. Circulation, 2004. 110(4): p. 460-5.

137. Abramson, J.L., et al., Moderate alcohol consumption and risk of heart failure among older persons. JAMA, 2001. 285(15): p. 1971-7.

138. Bryson, C.L., et al., The association of alcohol consumption and incident heart failure: the Cardiovascular Health Study. J Am Coll Cardiol, 2006. 48(2): p. 305-11.

139. Djousse, L. and J.M. Gaziano, Alcohol consumption and risk of heart failure in the Physicians' Health Study I. Circulation, 2007. 115(1): p. 34-9.

140. Djousse, L. and J.M. Gaziano, Alcohol consumption and heart failure in hypertensive US male physicians. Am J Cardiol, 2008. 102(5): p. 593-7.

141. Walsh, C.R., et al., Alcohol consumption and risk for congestive heart failure in the Framingham Heart Study. Ann Intern Med, 2002. 136(3): p. 181-91.

142. Padilla, H., J. Michael Gaziano, and L. Djousse, Alcohol consumption and risk of heart failure: a meta-analysis. Phys Sportsmed, 2010. 38(3): p. 84-9.

143. Klatsky, A.L., et al., Alcohol drinking and risk of hospitalization for heart failure with and without associated coronary artery disease. Am J Cardiol, 2005. 96(3): p. 346-51.

144. Urbano-Marquez, A., et al., The effects of alcoholism on skeletal and cardiac muscle. N Engl J Med, 1989. 320(7): p. 409-15.

145. Manolio, T.A., et al., Relation of alcohol intake to left ventricular mass: The Framingham Study. J Am Coll Cardiol, 1991. 17(3): p. 717-21.

146. Lazarevic, A.M., et al., Early changes in left ventricular function in chronic asymptomatic alcoholics: relation to the duration of heavy drinking. J Am Coll Cardiol, 2000. 35(6): p. 1599-606.

147. Figueredo, V.M., et al., Chronic alcohol-induced changes in cardiac contractility are not due to changes in the cytosolic Ca2+transient. Am J Physiol, 1998. 275(1 Pt 2): p. H122-30.

148. Zhang, B., et al., Cardiac overexpression of insulin-like growth factor 1 attenuates chronic alcohol intake-induced myocardial contractile dysfunction but not hypertrophy: Roles of Akt, mTOR, GSK3beta, and PTEN. Free Radic Biol Med, 2010. 49(7): p. 1238-53.

149. Nicolas, J.M., et al., The effect of controlled drinking in alcoholic cardiomyopathy. Ann Intern Med, 2002. 136(3): p. 192-200. 
150. Djousse, L., et al., Long-term alcohol consumption and the risk of atrial fibrillation in the Framingham Study. Am J Cardiol, 2004. 93(6): p. 710-3.

151. Mukamal, K.J., et al., Alcohol consumption and risk and prognosis of atrial fibrillation among older adults: the Cardiovascular Health Study. Am Heart J, 2007. 153(2): p. 260-6.

152. Mukamal, K.J., et al., Alcohol consumption and risk of atrial fibrillation in men and women: the Copenhagen City Heart Study. Circulation, 2005. 112(12): p. 1736-42.

153. Kodama, S., et al., Alcohol consumption and risk of atrial fibrillation: a metaanalysis. J Am Coll Cardiol, 2011. 57(4): p. 427-36.

154. Dietary Guidelines for Americans. 2005, United States Department of Agriculture and United States Department of Health and Human Services: Washington, DC. p. 43-46.

155. Gaziano, J.M., et al., Light-to-moderate alcohol consumption and mortality in the Physicians' Health Study enrollment cohort. J Am Coll Cardiol, 2000. 35(1): p. 96-105.

156. Keil, $\mathrm{U}$., et al., The relation of alcohol intake to coronary heart disease and allcause mortality in a beer-drinking population. Epidemiology, 1997. 8(2): p. 150-6.

157. Doll, R., et al., Mortality in relation to consumption of alcohol: 13 years' observations on male British doctors. BMJ, 1994. 309(6959): p. 911-8.

158. Renaud, S.C., et al., Alcohol and mortality in middle-aged men from eastern France. Epidemiology, 1998. 9(2): p. 184-8.

159. Yuan, J.M., et al., Follow up study of moderate alcohol intake and mortality among middle aged men in Shanghai, China. BMJ, 1997. 314(7073): p. 18-23.

160. Thun, M.J., et al., Alcohol consumption and mortality among middle-aged and elderly U.S. adults. N Engl J Med, 1997. 337(24): p. 1705-14. 


\section{Figure Legends}

Figure 1: Relationship between total mortality and alcohol intake extracted from 56 curves using fixed- and random-effects models. (With permission from Di Castelnuovo, A et al. Arch Intern Med, 2006)[8]

Figure 2: Alcohol consumption in relation to cardiovascular mortality in cardiovascular disease patients. (With permission from Costanzo, S et al. J Am Coll Cardiol, 2010)[9]

Figure 3: Beneficial mechanisms of light to moderate alcohol consumption

Table 1: Studies showing beneficial effects of light to moderate alcohol consumption on cardiovascular or all-cause mortality

Table 2: Studies showing neutral effects of light to moderate alcohol consumption on cardiovascular or all-cause mortality 
TABLE 1

Study/Cohort

Physicians' Health

Study[155]

National Health Interview

Survey[12]

Framingham Heart

Study[11]

German Study[156]

British physicians [157]

French Study[158]

Chinese men[159]

Cancer Prevention Study II [160]

Japanese Study[16]

Women's Health

Study[17]

Women's Health

Initiative[19]
Participants (n) Follow-up

89,299

245,207

4,745

2,084

12,321

34,014

18,244

490,000

309,082

26,399

116,186

5 years

24 years

8 years

13 years

6.7 years

9 years

12.4 years

12.2 years

8 years
All-cause (or) CHD mortality

Inverse association

Inverse association

U-shaped

U-shaped

U-shaped

10-15 years

U-shaped

U-shaped

J-, U- or L-shaped

J- or U-shaped

J-shaped

Inverse association 
TABLE 2

\begin{tabular}{|c|c|c|c|c|c|}
\hline Study & Cohort & $\begin{array}{l}\text { Participants } \\
\text { (n) }\end{array}$ & Follow-up & $\begin{array}{l}\text { All-cause (or) } \\
\text { CHD } \\
\text { mortality }\end{array}$ & Comment \\
\hline $\mathrm{NHEFS}^{[41]}$ & $\begin{array}{l}\text { African- } \\
\text { American }\end{array}$ & 2,054 & 19 years & No benefit & None \\
\hline Hart et al. ${ }^{[42]}$ & Scottish & 5,766 & 21 years & No benefit & $\begin{array}{l}\text { Increased } \\
\text { mortality with } \\
>22 \text { drinks/wk }\end{array}$ \\
\hline INTERHEART T $^{[43]}$ & $\begin{array}{l}\text { Asian- } \\
\text { Indian }\end{array}$ & 4,465 & N/A & N/A & $\begin{array}{l}\text { No inverse } \\
\text { effect on CHD, } \\
\text { may be harmful }\end{array}$ \\
\hline Schooling et al. ${ }^{[45]}$ & Chinese & 54,090 & 4.2 years & No benefit & None \\
\hline Zhou et al. ${ }^{[46]}$ & Chinese & 1,476 & N/A & No benefit & $\begin{array}{l}\text { Moderate to } \\
\text { heavy alcohol } \\
\text { intake was } \\
\text { detrimental for } \\
\text { CHD }\end{array}$ \\
\hline Deev et al. ${ }^{[4]]}$ & Russian & 4,153 & 13 years & No benefit & None \\
\hline $\begin{array}{l}\text { Strandberg et } \\
\text { al. }{ }^{[48]}\end{array}$ & Finnish & 1,808 & 29 years & No benefit & $\begin{array}{l}\text { Heavy alcohol } \\
\text { intake was } \\
\text { detrimental }\end{array}$ \\
\hline Shaper et al. ${ }^{[49]}$ & British & 7,169 & 12.8 years & No benefit & $\begin{array}{l}>/=3 \text { drinks } \\
\text { per day } \\
\text { detrimental }\end{array}$ \\
\hline
\end{tabular}

N/A - Not available 
Figure 1

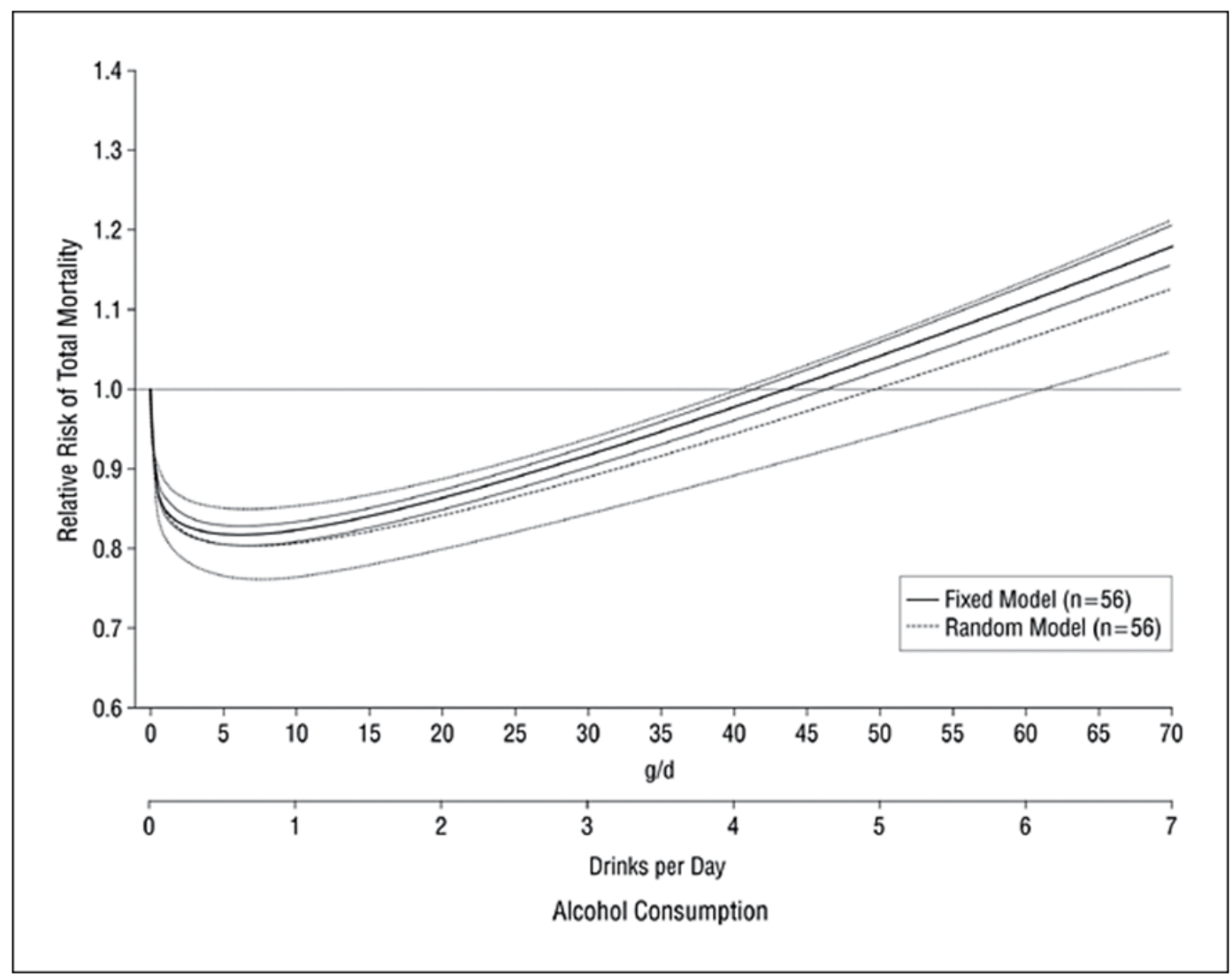


Figure 2

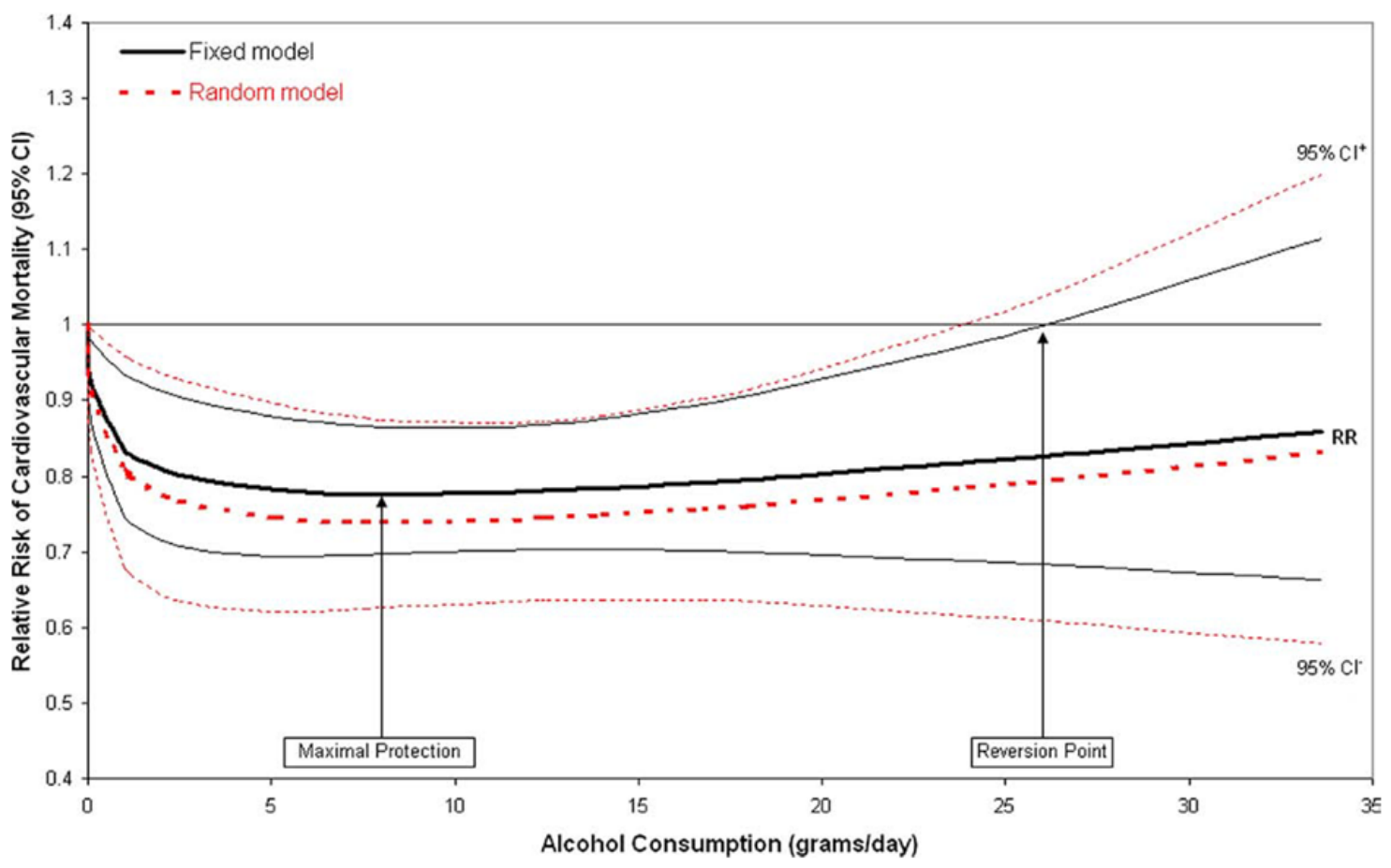


Figure 3

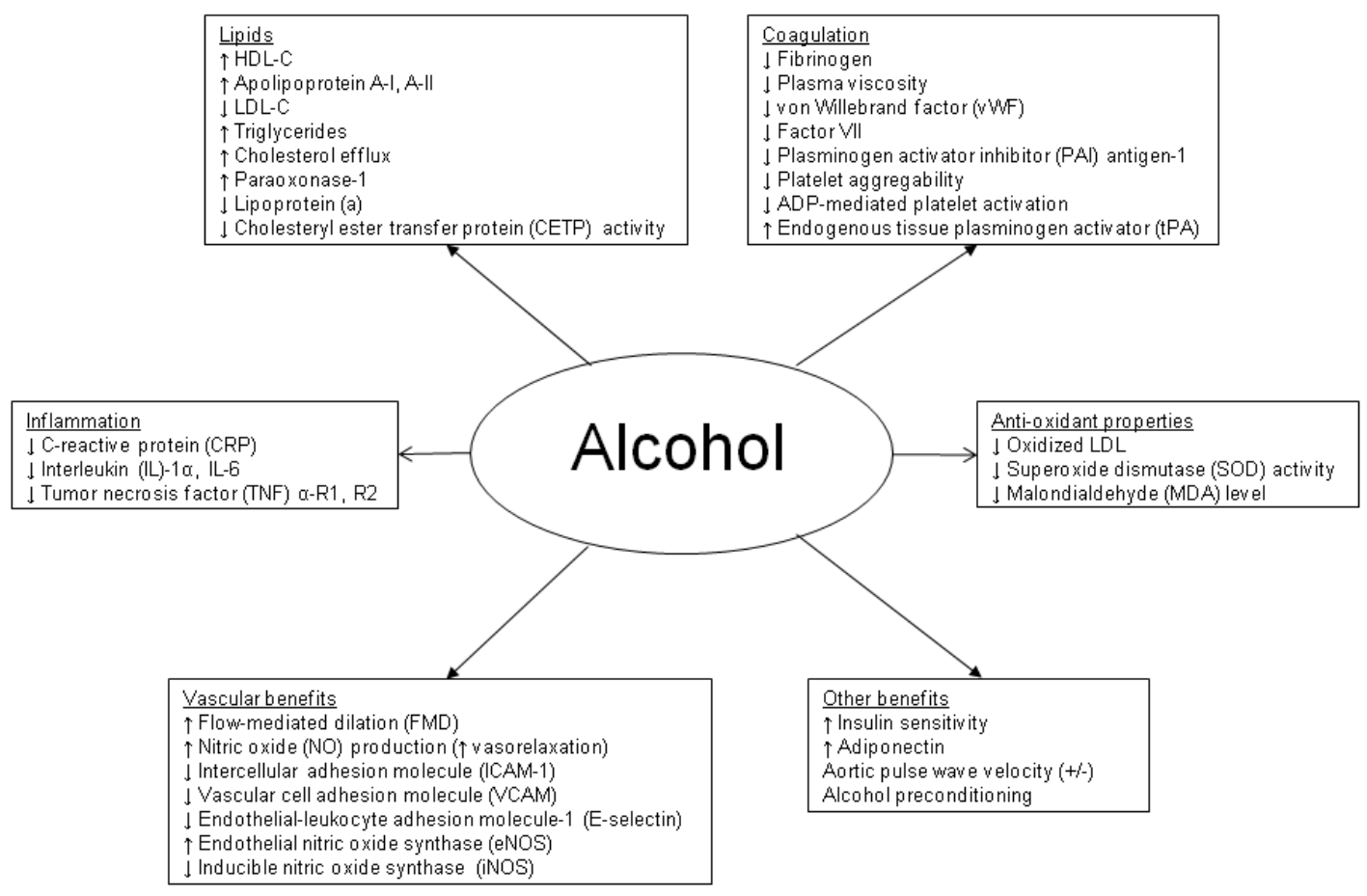

